Cross-sectional study

\section{Staffing and nurse-perceived quality of care}

10.1136/eb-2016-102478

Pamela B de Cordova

Rutgers School of Nursing, Newark, New Jersey, USA

Correspondence to: Professor Pamela B de Cordova, Rutgers School of Nursing, 180 University Avenue, Room 244, Newark, NJ 07102, USA; pam.decordova@rutgers.edu

Commentary on: Cho E, Lee NJ, Kim EY, et al. Nurse staffing level and overtime associated with patient safety, quality of care, and care left undone in hospitals: a cross-sectional study. Int J Nurs Stud 2016;60:263-71.

\section{Implications for practice and research}

- Nursing staff and administrators should be aware of the extensive evidence supporting the association of staffing and work hours on patient safety.

- Researchers should evaluate existing staffing policy legislations nationally and internationally to determine strengths and weaknesses of these approaches.

\section{Context}

For well over a decade, research examining the effect of nurse staffing on quality outcomes has grown extensively worldwide. As a result of these rigorous studies, the association between nurse staffing and patient safety has been established. Researchers are continuing to expand the context of nurse staffing literature by including factors such as the nurse's work environment, educational preparation and work hours in their analyses. This study by Cho and colleagues examines nurse staffing and overtime on nurse-perceived patient safety and care left undone for patients in South Korea.

\section{Methods}

The purpose of the study was to explore the associations between nurse staffing and overtime with nurse-perceived patient safety and care left undone. This study fills an important gap as the authors noted that nurse staffing research has been conducted predominantly in Western countries -not in Asian countries, such as South Korea.

The researchers used a cross-sectional survey from the International Hospital Outcomes Study (IHOS). There were 3037 registered nurses' (RNs) responses representing 51 hospitals that were analysed. Outcomes included measures for patient safety, quality of care and care left undone. To operationalise these outcomes, Cho and colleagues used nurse-perceived responses. Using the IHOS allowed the researchers to directly compare South Korean results with other countries that used this instrument. Multilevel logistic regression models accounting for clustering of nurses in hospitals were used to predict the nurse-perceived safety of the hospital unit and care left undone.

\section{Findings}

Approximately 65\% $(n=1959)$ of RNs reported the quality of care on their units as fair or poor, and $82 \%(n=2450)$ reported that one or more necessary activities were left undone due to lack of time. An increase of one patient per nurse was associated with a $2 \%$ increase in the odds of a nurse rating the unit's patient safety as poor $(\mathrm{OR}=1.02$, 95\% CI 1.01 to 1.04 ) and a $3 \%$ increase in the odds of care left undone $(\mathrm{OR}=1.03,95 \%$ CI 1.01 to 1.05). If the RNs worked overtime, the odds of the nurse rating patient safety as poor increased by $88 \%$.

\section{Commentary}

Cho and colleagues found that as patients per nurse and nurse work hours increase, so does the perception of reduced patient safety. This approach of using nurse reporting of staffing has been validated previously and has the advantage of obtaining the views of nurses who are providing direct patient care. ${ }^{1}$ Using a validated survey instrument, the authors were able to compare the nurse-perceived level of quality care in South Korea to the USA and Europe ${ }^{2}{ }^{3}$ and concluded that the level of perceived nurse quality is lower in South Korea than in other areas.

Cho and colleagues demonstrated that working overtime had an effect on perceived patient safety and care left undone. The unpredictability of patient care may contribute to a longer shift, as nurses feel a professional obligation to complete their work and ensure safe care prior to finishing their shift. Therefore, hospital administrators may not necessarily be requiring the nurse to work longer shifts, but rather the inherent desire of nurses to ensure that their patients are safe, and all care has been delivered prior to leaving work, may be the driving factor behind this overtime. It should be noted that the study found a high percentage of nurses were unable to complete nursing care even when working overtime.

This study reinforces the fact that educating nurses, administrators and policymakers about the importance of nurse staffing on quality care should continue. Although few would argue that reducing the number of nurses leads to better patient care, implementation of staffing legislation has been controversial. A logical first step would be for researchers to evaluate existing staffıng policies globally (if any even exist) and determine the effectiveness of such legislation. Conducting these policy analyses may assist in furthering the science and inform policymakers of a need for legislation concerning nurse staffing.

Funding This commentary was supported by the Agency for Healthcare Research and Quality (grant number 1 K08 HS024339-01A1).

Competing interests None declared.

Provenance and peer review Commissioned; internally peer reviewed.

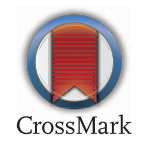

\section{References}

1. McHugh MD, Kelly LA, Sloane DM, et al. Contradicting fears, California's nurse-to-patient mandate did not reduce the skill level of the nursing workforce in hospitals. Health Aff (Millwood) 2011;30:1299-306.

2. Aiken LH, Sermeus W, Van den Heede K, et al. Patient safety, satisfaction, and quality of hospital care: cross sectional survey of nurses and patients in 12 countries in Europe and the United States. BMJ 2012;344:e1717.

3. Aiken LH, Sloane DM, Bruyneel L, et al. Nurses' reports of working conditions and hospital quality of care in 12 countries in Europe. Int J Nurs Stud 2013;50:143-53. 